\title{
Global Validity of the Boltzmann Equation for a Three- Dimensional Rare Gas in Vacuum
}

\author{
M. Pulvirenti ${ }^{\star}$ \\ Dipartimento di Matematica, Università dell'Aquila. L'Aquila, Italy
}

\begin{abstract}
We consider a system of $N$ hard spheres in the Boltzmann-Grad limit (i.e. $d \rightarrow 0, N \rightarrow \infty, N d^{2} \rightarrow \lambda^{-1}>0$, where $d$ is the diameter of the spheres). If $\lambda$ is sufficiently large, and if the joint distribution densities factorize at time zero, with the one particle distribution decaying sufficiently rapidly in space and velocities, we prove that the time evolved one-particle distribution converges for all times to the solution of the Boltzmann equation with the same initial datum. This result improves and is based on a previous paper [1], valid only in two dimensions.
\end{abstract}

1. In a recent paper [1] the validity of the Boltzmann equation has been proved for a cloud of gas of hard spheres in the two-dimensional vacuum. In the present paper we extend this result to the more physically relevant three-dimensional case.

A part of the techniques necessary to obtain the present result are contained in [1] to which we address the reader for motivations, general comments, further references and notation. We briefly review the result of [1] and explain why that approach fails in dimension three. Following the same notation, we denote by $\Gamma_{N}$ the phase space of a system of $N$ spheres of diameter $d$ in $\mathbb{R}^{v}(v=2,3), X$ $=\left\{x_{1} v_{1} \ldots x_{N} v_{N}\right\}, x_{i} v_{i} \in \mathbb{R}^{v} \times \mathbb{R}^{v}$ a phase point, $\phi_{t}^{d}$ the (almost everywhere defined) time evolution of the system, (for which $\phi_{t}^{d}(X)=\left\{x_{1}(t) v_{1}(t) \ldots x_{N}(t) v_{N}(t)\right\}$ is the trajectory of the phase point $X), \mu^{d}(d X)=\mu^{d}(X) d X$ an absolutely continuous, symmetric (in the exchange of particles), probability measure on $\Gamma_{N}$ at time zero, $\mu_{t}^{d}(X)$ the time evolved density, $f_{k, t}^{d} t \geqq 0,0<k \leqq N$ the joint distribution densities. Finally $S^{d}(t)$ is defined by:

$$
S^{d}(t) f_{k}^{d}\left(x_{1} v_{1} \ldots x_{k} v_{k}\right)=f_{k}^{d}\left(\phi_{-t}^{d}\left(\left\{x_{1} v_{1} \ldots x_{k} v_{k}\right\}\right)\right) .
$$

The following equation is satisfied by the family $f_{k, t}^{d}$ :

$$
f_{k, t}^{d}=S^{d}(t) f_{k}^{d}+\int_{0}^{t} d s S^{d}(t-s) C_{k, k+1}^{d} f_{k+1, s}^{d}
$$

* Partially supported by MPI and GNFM (CNR) 
where $f_{k}^{d}$ stands for $f_{k, 0}^{d}$ and $C_{k, k+1}^{d}$, the collision operator, is defined by formula (2.7) of [1] and will be recalled later.

The validity of Eq. (1.2) is proved in [1] and, in a more extended and natural way in [2], where also the history of the equation is reviewed.

In this paper we are interested in the asymptotic behavior of $f_{k, t}^{d}$ for large $N$, keeping fixed the product, $N d^{\nu-1}=\lambda^{-1}>0$.

Together with Eq. (1.2) we also consider the Boltzmann hierarchy (with initial datum $\left.f=\left\{f_{k}\right\}_{k=1 \ldots \infty}\right)$ :

where

$$
f_{k, t}=S(t) f_{k}+\int_{0}^{t} d s S(t-s) C_{k, k+1} f_{k+1, s}
$$

$$
\begin{gathered}
S(t) f_{k}\left(x_{1} v_{1} \ldots x_{k} v_{k}\right)=f_{k}\left(x_{1}-v_{1} t, v_{1} \ldots x_{k}-v_{k} t, v_{k}\right) \\
C_{k, k+1} f_{k+1}\left(x_{1} v_{1} \ldots x_{k} v_{k}\right)=\lambda^{-1} \sum_{j=1}^{k} \int d v_{k+1} \int_{n\left(v_{j}-v_{k+1}\right) \geqq 0} d n n \cdot\left(v_{j}-v_{k+1}\right) \\
f_{k+1}\left(x_{1} v_{1} \ldots x_{j} v_{j}^{\prime} \ldots x_{j} v_{k+1}^{\prime}\right)-f_{k+1}\left(x_{1} v_{1} \ldots x_{j} v_{j} \ldots x_{j} v_{k+1}\right) .
\end{gathered}
$$

Here, as usual, $n$ is a unitary vector and $v_{j}^{\prime}, v_{k+1}^{\prime}$ are the outgoing velocities after a collision with impact parameter $n$ and incoming velocities $v_{j}, v_{k+1}$.

The solution of Eq. (1.2) and the solution of Eq. (1.3) (if any) are expressed by the series (where $f_{t}^{d}=\left\{f_{k, t}^{d}\right\}_{k=1 \ldots \infty}, S^{d}(t) f^{d}=\left\{S^{d}(t) f_{k}^{d}\right\}_{k=1 \ldots \infty}, C^{d} f=\left\{\left(C^{d} f\right)_{k}\right\}_{k=1 \ldots \infty}$ $=\left\{C_{k, k+1}^{d} f_{k+1}\right\}_{k=1 \ldots \infty}$, and we use the convention $f_{k, t}^{d}=0$ if $k>N$ )

$$
\begin{gathered}
f_{t}^{d}=S^{d}(t) f^{d}+\sum_{n=1}^{\infty} \int_{0}^{t} d t_{1} \int_{0}^{t_{1}} d t_{2} \ldots \int_{0}^{t_{n-1}} d t_{n} S^{d}\left(t-t_{1}\right) C^{d} \ldots S^{d}\left(t_{n}\right) f^{d}, \\
f_{t}=S(t) f+\sum_{n=1}^{\infty} \int_{0}^{t} d t_{1} \int_{0}^{t_{1}} d t_{2} \ldots \int_{0}^{t_{n}-1} d t_{n} S\left(t-t_{1}\right) C \ldots S\left(t_{n}\right) f .
\end{gathered}
$$

The study of the behavior of $f_{t}^{d}$ in the Boltzmann-Grad limit $d \rightarrow 0, N \rightarrow \infty$, $N d \rightarrow \lambda^{-1}$ is of great conceptual interest. Actually $f_{t}^{d}$ is believed to converge to $f_{t}$ and a rigorous proof of this fact provides a proof of the validity of the Boltzmann equation itself. A first step in this direction was obtained by Lanford in a well known paper [3]. He proved the convergence of $f_{t}^{d}$ to $f_{t}$ in the Boltzmann-Grad limit for short times (but also in more general contexts than the case of a perturbation of the vacuum considered in [1] and in the present paper).

In [1] following the same Lanford strategy it was proved the convergence of $f_{t}^{d}$ to $f_{t}$ for all times but only for $d=2$. In fact it is possible to estimate both series (1.6) and (1.7) by means of absolutely convergent series by estimating each term in a suitable norm. Furthermore the convergence of the series (1.6) to the series (1.7) almost everywhere (in the limit $N \rightarrow \infty$ and $d$ chosen according to $N d^{v-1}=\lambda^{-1}$ ) follows by the convergence term by term of the series. Namely, rewriting the two series (1.6) and (1.7) in the form $(d \geqq 0)$ :

$$
\begin{aligned}
S^{d}(-t) f_{t}^{d}= & f^{d}+\sum_{n=1}^{\infty} \int_{0}^{t} d t_{1} \int_{0}^{t_{1}} d t_{2} \ldots \int_{0}^{t_{n-1}} d t_{n} \Omega^{d}\left(t_{1}\right) V^{d}\left(t_{1}\right) \Omega^{d}\left(t_{1}\right)^{-1} \ldots \\
& \Omega^{d}\left(t_{n}\right) V^{d}\left(t_{n}\right) \Omega^{d}\left(t_{n}\right)^{-1} f^{d},
\end{aligned}
$$


where

$$
\begin{aligned}
& \Omega^{d}(t)=S^{d}(-t) S(t), \\
& V^{d}(t)=S(-t) C^{d} S(t),
\end{aligned}
$$

and introducing the following norms:

$$
\begin{aligned}
\left\|f_{j}\right\|_{\beta, j} & =\operatorname{ess} \sup \left|f_{j}\left(x_{1} v_{1} \ldots x_{j} v_{j}\right)\right| \exp \beta \sum_{i=1}^{j}\left(x_{i}^{2}+v_{i}^{2}\right), \\
\|f\|_{\beta}^{z} & =\sup _{j} z^{-j}\left\|f_{j}\right\|_{\beta, j},
\end{aligned}
$$

it is possible to prove the following estimate (see Proportion 3.2, [1]):

$$
\begin{aligned}
\left\|V^{d}(t) f\right\|_{\beta^{\prime}}^{z^{\prime}} \leqq & C_{0} z \mid(\beta) \lambda^{-1}\left\|f_{j}\right\|_{\beta, j}\left(1+t^{\nu+1}\right)^{-1} \\
& \cdot\left\{\left(\ln z^{\prime} z^{-1}\right)+\sqrt{ }\left(\beta-\beta^{\prime}\right)\left(\ln z^{\prime} z^{-1}\right)\right\}
\end{aligned}
$$

for $1>d \geqq 0, \beta>\beta^{\prime}>0, z^{\prime}>z>0, l(\beta)=\sqrt{ } \max \left(\beta^{-v}, \beta^{-v+1}\right)$.

In the case of the Boltzmann hierarchy $(d=0)$, the estimate (1.13) is enough in order to obtain the convergence of the series (see Remark 2, p. 195 of [1]) and, moreover, a unique solution of Eq. (1.3) and, in particular, a unique (mild) solution of the Boltzmann equation in case of initial factorization. The argument is simple. Reminding that for the Boltzmann hierarchy $\Omega^{d}=1$, inserting the estimate (1.13) in the series (1.8) for $d=0$, after a reordering of times and an integration over all times (possible because of the factor $\left(1+t^{v+1}\right)^{-1}$ ) we are led to a geometric series converging for large $\lambda$. Roughly speaking this is same as the Lanford argument with the time replaced by $\lambda^{-1}$.

To bound the series (1.8) for $d>0$, we need to control $\Omega^{d}$ in the norm $\|\cdot\|_{\beta, j}$, and here the dimensionality of the physical space enters.

Defining $l(X)=\frac{1}{2} \sum_{i=1}^{j}\left(x_{i}^{2}+v_{i}^{2}\right)$, we arrive, after elementary considerations, at the formula:

$$
l\left(\phi_{t}^{d}(X)\right)=l\left(\phi_{t}(X)\right)+\sum_{i=1}^{k}\left(t_{i+1}-t_{i}\right)\left(y_{i}^{\prime}-y_{i}\right)\left(p_{i}^{\prime}-u_{i}^{\prime}\right)
$$

where $\phi_{t}^{d}(X)$ denotes the trajectory of a system of $j$ particles of diameter $d$ whose phase point at time zero is denoted by $X, \phi_{t}(X)$ the free motion for the same system, $t_{i}$ the collision times, $y_{i}^{\prime}, y_{i}$ the positions of the two particles at the $i^{\text {th }}$-collision, $p_{i}^{\prime}$ and $u_{i}^{\prime}$ the outgoing and incoming velocities of the particle in $y_{i}^{\prime}$ (see Proposition 3.1 of [1]).

Since the distributions for the $j$-particle system have a gaussian decay in the velocities, we may assume that the energy of the system $E$ is, at most, of order $N$. Thus in the worst case: (a single central collision between two particles with half energy)

$$
\begin{aligned}
l\left(\phi_{t}^{d}(X)\right) & =l\left(\phi_{t}(X)\right)+2 t d \sqrt{ } E \cong l\left(\phi_{t}(X)\right)+C t \sqrt{ } N d^{2} \\
& =l\left(\phi_{t}(X)\right)+C t \lambda^{-1 / 2} \sqrt{ } d^{3-v}
\end{aligned}
$$

for some positive constant $C$. Hence, for $j$ of the same order of $N$,

$$
\left\|\Omega^{d}(t)\right\|_{\beta, j} \cong \exp C 2 t \lambda^{-1 / 2} \sqrt{d^{3-v}}
$$


which means that $\Omega^{d}(t)$ is bounded, uniformly in $t \leqq T$, for a sufficiently small $d$, chosen according to $T$, if $v=2$. The $t$ dependence in Eq. (1.16), for $v=3$ does not allow us to integrate over all times, so that the argument fails.

The above considerations show that low probability events (a relevant part of the energy concentrated on a single particle undergoing a collision) make it difficult to control the series (1.8) for $d>0$, in terms of uniform norms.

In this paper we overcome this difficulty by introducing a fictitious dynamics which ignores the high energy collisions so that the approach in [1] applies also in dimension three. In addition we estimate the probability that the true dynamics differs from the new one and find that this is negligible in the Boltzmann-Grad limit. As a consequence we prove a validity theorem for the Boltzmann equation in a slightly weaker form: the $f_{k, t}^{d}$ will converge to $f_{k, t}$ weakly and not almost everywhere as in [3] and [1]. However this does not seem a physically relevant point.

2. In this section we always assume the dimension of the physical space to be three.

We introduce the (reversible) dynamics, denoted by $\phi_{t}^{d}$, for a system of hard spheres, according to the following definition. $\phi_{t}^{d}$ coincides with $\phi_{t}^{d}$ unless two particles collide with enrgy $E(i, j)=1 / 2\left(v_{i}^{2}+v_{j}^{2}\right)$ larger than $\sqrt{ } N$. In that case they are not elastically reflected, but they go ahead freely. Obviously the phase space of the system, $\Gamma_{N}$, is slightly larger than $\Gamma_{N}$, since overlapping of a pair of particles with large energy are allowed. We underline all the objects which refer to this new dynamics: e.g. $\underline{\mu}_{t}^{d}, f_{k, t}^{d}, \underline{S}^{d}(t), \ldots$ Exactly along the same lines leading to Eq. (1.2) (see [1] and [2]) we have:

$$
\underline{f}_{k, t}^{d}=\underline{s}^{d}(t) f_{k}^{d}+\int_{0}^{t} d s \underline{S}^{d}(t-s) \underline{C}_{k, k+1}^{d} \underline{f}_{k+1, s}^{d},
$$

where

$$
\begin{gathered}
\underline{C}_{k, k+1}^{d} f_{k+1}\left(x_{1} v_{1} \ldots x_{k} v_{k}\right)=(N-k) d^{v-1} \sum_{j=1}^{k} \int d v_{k+1} \int d n n \cdot\left(v_{j}-v_{k+1}\right), \\
f_{k+1}\left(x_{1}, v_{1} \ldots x_{j}, v_{j} \ldots x_{j}+d n, v_{k+1}\right) \chi(E(j, k+1) \leqq \sqrt{ } N),
\end{gathered}
$$

and $\chi$ (something) denotes the characteristic function of the set in which something happens. We remind that $C_{k, k+1}^{d}$ is defined as $\underline{C}_{k, k+1}^{d}$ provided that the indicator $\chi$ is neglected.

By Eq. (1.14) we obtain:

$$
l\left(\phi_{t}^{d}(X)\right) \leqq l\left(\phi_{t}(X)\right)+(2 \sqrt{ } 2) t N^{1 / 4} d,
$$

so that, fixed arbitrarily $T>0$ and $\varepsilon>0$, for $N$ sufficiently large (depending on $T, \lambda, \varepsilon$ ), we have (for $t<T$ ):

$$
\left\|\underline{\Omega}^{d}(t)\right\|_{\beta, j} \leqq \exp \varepsilon, \quad\left\|\Omega^{d}(t)^{-1}\right\|_{\beta, j} \leqq 1 .
$$

The last inequality follows because the last term in Eq. (1.14) is positive.

The following Theorem is consequence of the arguments in [1].

Theorem 1. Assume, for the joint distribution densities at time zero, the following hypotheses: 
i) They are continuous functions in $\left(\mathbb{R}^{3} \times \mathbb{R}^{3}\right)_{\neq}^{j, d}=\left\{X|| x_{i}-x_{k} \mid>d\right.$ for $i \neq k, i$, $k=1, \ldots, j d \times 0\}$, vanishing on the boundary of $\left(\mathbb{R}^{3} \times \mathbb{R}^{3}\right)_{\neq}^{\jmath, d}$. There exists an infinite sequence of functions $\left\{f_{j}\right\}_{j=1 \ldots \infty}$ continuous on $\left(\mathbb{R}^{3} \times \mathbb{R}^{3}\right)_{\neq}^{j, 0}$ such that

$$
\lim _{d \rightarrow 0} f_{j}^{d}=f_{j} \text { for all uniformly on compact sets of }\left(\mathbb{R}^{3} \times \mathbb{R}^{3}\right)_{\neq}^{j, 0} \text {. }
$$

ii) There exists $C_{1}>0$ such that, for $z_{0}>0$ and $\beta_{0}>1$

$$
\left\|f^{d}\right\|_{\beta_{0}} z_{0} \leqq C_{1} \text {. }
$$

Then, there exists an increasing positive function $\gamma=\gamma\left(\beta_{0}\right)$ such that, if $\lambda^{-1} z_{0}<\gamma\left(\beta_{0}\right)$, then:

$$
\lim _{d \rightarrow 0^{-}}^{d} f_{j, t}=f_{j, t} \text { a.e. }
$$

Moreover, for any $T>0, z=z_{0} e, \beta=\beta_{0}-1$ and $d$ sufficiently small,

$$
\sup _{t<T}\left\|\underline{f}_{t}^{d}\right\|_{\beta}^{z}<\text { const. }
$$

Proof. In what follows it is convenient to extend $f_{k, t}^{d}$ (as well as $\underline{f}_{k, t}^{d}$ ) to the whole space $\left(\mathbb{R}^{3} \times \mathbb{R}^{3}\right)^{k}$, by putting $f_{k, t}^{d}(X)=0\left(\underline{f}_{k, t}^{d}(X)=0\right)$ if $X$ is such that $\left|x_{i}-x_{j}\right|<d$ for some $i \neq j$ (in case $E(i, j) \leqq \sqrt{ } N$ ).

The estimate (1.13) is obviously valid also for $\underline{V}^{d}(t)$ and hence, by iterating the estimates (1.13) and (2.6) $n$ times for the $n^{\text {th }}$ term of the series (1.8), with $\ln$ $z_{j} / z_{j-1}=n^{-1}$ and $\beta_{j-1}=\beta_{j}+n^{-1} j=1 \ldots n$, we obtain (after reordering the times and integrating up to infinity) for an arbitrary fixed $T>0$ and $d$ sufficiently small:

$$
\| n^{\text {th }} \text { term }\left\|_{\beta}^{z} \leqq f^{d}\right\|_{\beta_{0}}^{z_{0}}\left(C_{2} n z_{0} \lambda^{-1} l(\beta)\right)^{n} e^{\varepsilon n}(n !)^{-1}
$$

with $z=z_{0} e, \beta=\beta_{0}-1$ and $C_{2}>0$.

Therefore the series (1.8) is converging for $\lambda^{-1}$ small, uniformly in $N$ and $d$, (this implies (2.9). The statement (2.8) follows by a direct inspection of the convergence term by term (see [3] and also [4] for a revisited version).

We are now in position to prove the main result of this paper.

Theorem 2. Under the hypotheses i) and ii) of Theorem 1, if $\lambda^{-1} z_{0}<\gamma\left(\beta_{0}\right)$,

$$
\lim _{d \rightarrow 0} \int f_{k, t}^{d} g=\int f_{k, t} g
$$

for all $k>0$ and all bounded continuous functions $g:\left(\mathbb{R}^{3} \times \mathbb{R}^{3}\right)^{k} \rightarrow \mathbb{R}$.

Proof. We have:

$$
\int\left(f_{k, t}^{d}-f_{k, t}\right) g=\int\left(\underline{f}_{k, t}^{d}-f_{k, t}\right) g+\int\left(f_{k, t}^{d}-\underline{f}_{k, t}^{d}\right) g
$$

The first term in the right-hand side of (2.12) goes to zero as a consequence of Theorem 1. In fact the integrand vanishes almost everywhere and is uniformly bounded by an integrable function (by 2.9).

Denoting by $G:\left(\mathbb{R}^{3} \times \mathbb{R}^{3}\right)^{N} \rightarrow \mathbb{R}$

$$
G(X)=g\left(x_{1}, v_{1} \ldots x_{k} v_{k}\right) \text { for } X=\left(x_{1}, v_{1} \ldots x_{N} v_{N}\right)
$$


we have, by Liouville Theorem (which holds also for $\phi_{t}^{d}$ )

$$
\begin{gathered}
\int\left(f_{k, t}^{d}-\underline{f}_{k, t}^{d}\right) g=\int d X G(X)\left\{\underline{\mu}_{t}^{d}(X)-\mu_{t}^{d}(X)\right\} \\
=\int d X\left\{G\left(\phi_{t}^{d}(X)\right)-G\left(\phi_{t}^{d}(X)\right)\right\} \mu_{0}^{d}(X) .
\end{gathered}
$$

Defining:

$$
\begin{gathered}
B_{N}=\bigcup_{i \neq j} B_{N}(i, j), \\
B_{N}(i, j)=\left\{X|| x_{i}-x_{j} \mid=d, \quad E(i, j)>\sqrt{ } N, \quad\left(v_{i}-v_{j}\right) \cdot\left(x_{i}-x_{j}\right)>0\right\}, \\
B_{N}(T)=\bigcup_{0 \leqq t \leqq T} \phi_{-t}^{d}\left(B_{N}\right),
\end{gathered}
$$

we realize that $\left\{G\left(\phi_{t}^{d}(X)\right)-G\left(\phi_{t}^{d}(X)\right)\right\}$ is zero unless $X \in B_{N}(T)$. Therefore the righthand side of Eq. (2.14) is bounded by $2\|g\|_{\infty} \mu_{0}^{d}\left(B_{N}(T)\right)$.

To estimate the $\mu_{0}^{d}$-measure of the "bed set" $B_{N}(T)$ we take advantage by the following, so-called, special flow representation.

Consider the following map

$$
\begin{aligned}
\Psi: B_{N}(T) & \rightarrow B_{N}(T), \quad \Psi(X)=(Y, t) \quad \text { if } \phi_{-t}^{d} Y=X, \quad Y \in B_{N}, \\
B_{N}(T) & =\left\{(Y, t) \mid Y \in B_{N}, 0 \leqq t<\varphi(Y)\right\}, \\
\varphi(Y) & =\sup \left\{t>0 \mid \phi_{-t}^{d} Y \notin B_{N}\right\} .
\end{aligned}
$$

Then $\Psi$ is an almost everywhere defined one to one bimeasurable map.

On the Borel sets of $B_{N}(i, j)$ we defined the following measure:

$$
\begin{aligned}
d \sigma_{i j}(Y) & =d x_{1} \ldots d x_{j-1} d y_{j} d x_{j+1} \ldots d x_{N} d v_{1} \ldots d v_{N} n_{i} \cdot\left(v_{i}-v_{j}\right) . \\
\chi(E(i, j) & >\sqrt{ } N),
\end{aligned}
$$

where $d y_{j}$ is the Lebesgue measure of the sphere of center $x_{i}$ and radius $d$ and $n_{i j}=\left(x_{j}-x_{i}\right) /\left|x_{j}-x_{i}\right|$. Denoting by $d \sigma$ the Borel measure on $B_{N}$ whose restriction on $B_{N}(i, j)$ is $d \sigma_{i j}$, we have the following formula:

$$
\int_{B_{N}(T)} F(X) d X=\int_{B_{N}} d \sigma(Y) \int_{0}^{\varphi(Y) \wedge T} d t F\left(\Psi^{-1}(Y, t)\right),
$$

where $g$ is any bounded measurable function, and

$$
\varphi(Y) \wedge T=\min (\varphi(Y), T) .
$$

By virtue of the estimate (2.9) we obtain, for $F=\mu_{0}^{d}$ :

$$
\begin{aligned}
\mu_{0}^{d}\left(B_{N}(T)\right) \leqq & \int_{0}^{T} d t \int_{B_{N}} d \sigma(Y) \underline{\mu}_{t}(Y) \\
\leqq & \sum_{i \neq j} \int_{0}^{T} d t \int \underline{f}_{2, t}^{d}\left(x_{i}, v_{i}, y_{j}, v_{j}\right) \chi(E(i, j)>\sqrt{ } N) \\
& d x_{i} d y_{i} d v_{i} d v_{j} n_{i j} \cdot\left(v_{j}-v_{i}\right) \\
\leqq & A(T) N^{2} d^{2} \exp \{-H(T) \sqrt{ } N\}
\end{aligned}
$$


where $A(T)$ and $H(T)$ are two positive constants not depending of $N$. Therefore $\mu_{0}^{d}\left(B_{N}(T)\right)$ goes to zero in the Boltzmann-Grad limit and this achieves the proof.

\section{References}

1. Illner, R., Pulvirenti, M.: Global validity of the Boltzmann equation for a two-dimensional rare gas in a vacuum. Commun. Math. Phys. 105, 189-203 (1986)

2. Illner, R., Pulvirenti, M.: A derivation of the BBGKY-hierarchy for hard sphere particle systems, Preprint University of Victoria (1985). To appear in Transp. Theory Stat. Phys.

3. Lanford, O. III: Time evolution of large classical systems. Moser, E. J. (ed.) Lecture Notes in Physics. Vol. 38, pp. 1-111. Berlin, Heidelberg, New York: Springer 1975

4. Shinbrot, M.: Math Methods Appl. Sci. 6, 539-549 (1984)

Communicated by J. L. Lebowitz

Received January 21, 1987 
